\title{
Does Childhood Trauma Lead to Sustained Hallucinations in Preschool-Aged Children? A Case Report
}

\author{
Mahin Eslami Shahrbabaki, ${ }^{1}$ and Parisa Divsalar ${ }^{2, *}$ \\ ${ }^{1}$ Psychiatry Department, Neuroscience Research Centre, Institute of Neuropharmacology, Afzalipour Medicine School, Shahid Beheshti Hospital, Kerman University of \\ Medical Sciences, Kerman, IR Iran \\ ${ }^{2}$ Bam University of Medical Sciences, Bam, IR Iran \\ "Corresponding author: Parisa Divsalar, Bam University of Medical Sciences, Bam, IR Iran. E-mail: pdivsalar@yahoo.com
}

Received 2016 February 05; Revised 2016 July 08; Accepted 2016 August 30.

\begin{abstract}
Introduction: Hallucinations in children have not typically been viewed as synonymous with psychosis. They can even be part of normal development or can be associated with other psychiatric disorders. These hallucinations are sometimes referred to as non-psychotic hallucinations. An association between childhood trauma and psychosis has been demonstrated several times.

Case Presentation: A six-year-old child was analyzed as a follow-up study over a period of three years by a child and adolescent psychiatrist. During the study, he was referred to the psychiatric hospital three times for aggression and hallucinations. Despite his several admissions to the psychiatric hospital and receipt of vigorous treatment, he maintained clear and sustained hallucinations that never diminished. At the last outpatient follow-up, his mother revealed physical abuse by his father. Laboratory tests, magnetic resonance imaging, and electroencephalography results were unremarkable.

Conclusions: Based on our experience and knowledge, this child, who has had three years of sustained and non-psychotic hallucinations, is a rare case. In addition, this case proves that the diagnostic complexity of hallucinations in children of preschool age requires a high level of precision and thoroughness. That is, these hallucinations may be associated with adversities experienced by the child. This article discusses how trauma may manifest in characteristic symptoms of psychosis, particularly hallucinations.
\end{abstract}

Keywords: Child, Hallucination, Psychosis, Trauma

\section{Introduction}

The clinical phenomenon of auditory hallucinations is defined as auditory perceptions in the absence of clear external stimuli (1). In adult psychiatric literature, the presence of auditory hallucinations is viewed as being synonymous with psychotic disorders, which represent serious psychopathology and unfavorable prognosis. However, auditory hallucinations in children and adolescents are rather less significant diagnostically, can be part of normal development, or can be associated with non-psychotic psychopathology. In addition, a range of mental health problems may produce auditory hallucinations. For instance, auditory hallucinations have been described in children and adolescents with conduct and emotional disorders, difficulties in coping, bereavement, affective syndromes, migraine, anxiety, and adjustment disorders (2-5).

The term "psychosis" has several definitions, the narrowest being delusions or prominent hallucinations in the absence of insight (6). Other definitions comprise the symptoms of schizophrenia, such as disorganized speech, disorganized or catatonic behavior, loss of ego boundaries, and impaired reality testing. The DSM-IV-TR does not address the clinical phenomenon of non-psychotic hallucinations in children. Accordingly, Garralda's concept of non- psychotic hallucinations in children is used in this article $(2,7)$.

Epidemiological research has shown that hallucinations and delusions, the classic symptoms of psychosis, are much more common in the general population than actual psychotic disorders. Longitudinal studies have shown that psychotic symptoms in adolescence may increase the risk of psychotic disorder in adulthood. However, there have been few studies on the clinicopathological significance of psychotic symptoms in children (8).

Environmental stress during childhood plays an important role in raising the risk of psychosis (9). In the last decade, association between childhood trauma and psychosis has been demonstrated several times (10). Population-based studies have demonstrated that the association between adversity and psychosis remains at large when confounders' parameters, including family history of psychosis, age, sex, ethnicity, marital status, other psychiatric diagnoses, education, neuroticism, and substance use are controlled (11). Although the relationship between exposure to trauma and psychosis is authorized within the articles, evidence is lacking regarding the precise psychological mechanisms by which trauma impacts on the occurrence of psychosis (12). 
This report presents a child who was prospectively studied as part of a three-year follow-up study. Despite receiving treatment, his auditory, visual, and tactile hallucinations never decreased. However, his family was satisfied with his mental health improvement.

\section{Case Presentation}

A six-year-old boy presented with problems such as agitation, aggression, wrangle, high dependency on his mother, and excuse-making that had continued for three years. He had been observed talking to images on the wall and was arrogant and sensitive to any noise in the house.

At times, he pulled at his clothes until they ripped apart. At the same time, he claimed "a black creature hits me in my head and then hides under the ground." He was irritable, was known to bite his sister and hit his mother, and was very sensitive about his personal property, to the point that no member of his family was allowed to touch his belongings. As a result of his aggressiveness, his father had fought with him and, sometimes, had beaten him. The child underwent outpatient treatment at a mental hospital and, despite his diagnosis of dystonia, his mother discontinued his medications without informing the doctor. He had been confined three times in the previous two years in the child and adolescent ward of the mental hospital, where he was visited by the child and adolescent psychiatrist and her residents.

During his hospitalization, he suffered from insomnia and restlessness, awakening in the middle of the night and claiming that a person alike his mother beat him and went under the ground. He said to his mother "you are not my real mother, you killed my mother, and I want my own mother." He also insisted that he saw a boy with a blue face and yellow hat wearing a long black outfit. This boy reportedly annoyed the child, pulled his hair, owned a knife, and intended to kill him.

In terms of his personal history, the child was born by Caesarean section, due to post-term pregnancy and oligohydramnios. His course of development and growth was normal. He has Clear history of convulsion or head trauma. He had a history of enuresis, separation anxiety, and fear of animals. At night, he slept next to his mother and placed his hand on his mother's chest. Due to the nuisance that he could be, he was frequently verbally abused by relatives who told him, in the presence of his parents, that they would cut off his ears and put his head into the spinning ceiling fan blades.

The boy lived as part of a family of four, including his sister, mother, and father. The family was of low socioeconomic status. There was no reported history of any mental problems among his first-degree relatives. Only a dubious history, without reliable evidence, of mental illness in the patient's cousin was reported.

The electrochemistry, blood and urine tests, and thyroid and liver function tests were normal. Electroencephalography (EEG) results were normal. The patient underwent neurologic counselling, and the brain MRI showed normal results. Wilson's disease was also ruled out, following a visit to the ophthalmologist.

Initially, the patient was diagnosed with PTSD and obsessive compulsive disorder (OCD), while the potential of bipolar disorder was dismissed. During his first in-patient stay, he was given $1 \mathrm{mg}$ of risperidon at night and $25 \mathrm{mg}$ of sertraline twice each day. Parental education and guidance (i.e., principles of behavior modification) were provided. During his second in-patient stay, he was given $1 \mathrm{mg}$ of risperidon three times each day, $300 \mathrm{mg}$ of lithium twice each day, $2 \mathrm{mg}$ of biperidin daily, and $25 \mathrm{mg}$ of sertraline twice each day. During his last in-patient admission, the risperidon was increased to $2 \mathrm{mg}$, three times each day, and the lithium was replaced with $200 \mathrm{mg}$ of valproate sodium three times each day. His parents attended parenting program education. The patient was discharged each time after a partial remission, while the hallucinations continued.

The patient had regular outpatient visits with the child and adolescent psychiatrist. For the next five months, although the visual and tactile hallucinations continued, he maintained a partial remission.

After unauthorized withdrawal of drugs by his mother at the sixth month, the patient's symptoms, such as restlessness, insomnia, aggression, and the thought that someone was going to harm him recurred. He was reported as saying “Arshia tells me 'throw your shoe.' He is older than me and bothers me. Dorsa is here too. Pulls my hair." According to his mother, he had enuresis and blinked rapidly(i.e., a possible tic disorder). The patient underwent treatment consisting of a daily dose of $4 \mathrm{mg}$ of risperidon, $5 \mathrm{mg}$ of olanzapine at night, and a daily dose of 400mg of valproate sodium. After two weeks, his sleeping pattern improved and his restlessness disappeared, but his aggression remained the same. At the next follow-up, his mother revealed "he was often beaten to death by his father." She reported that she had been threatened not to talk about the case. The patient was reported as saying "Arshia does not give me his marble and harasses me. He pulls my hair, hits me, and hides rapidly." In addition, the patient did not like to leave the house, did not allow his family members to touch his property, and was still agitated.

\section{Discussion}

Based on the description of the patient, we can discern that the diagnostic complexity of hallucinations in chil- 
dren of preschool age requires a particularly high level of precision. Hallucinations in children receive serious attention from parents and clinicians; however, they may not necessarily be symptoms of serious mental illness (13).

The child in this study had auditory, visual, and tactile hallucinations which were frightening and sometimes occurred in the middle of the night. In his case series, Pao evaluated ten children, during a period of 20 months, who presented with visual and tactile hallucinations that subsided within few weeks (14). However, in our case, the hallucinations never ceased. In Pao's study, the hallucinations were anxiety-based, and all of the analyzed children had grown up in families of low to median socioeconomic status. Pao considered the hallucinations as benign, phobic hallucinations that were self-limited and had been reported to occur only in preschool- to young school-aged children (14). The child in this study was also from a family of low socioeconomic status, had separation anxiety disorder and a specific phobia, and his hallucinations were anxiety-based.

The boy in our study had frequent fights with his sister and sometimes behaved impulsively. It appeared that his sister received more attention from their parents than he did, which may apparently justify his aggression. On the other hand, he displayed an attempt to place the blame for his negative behavior (e.g., beating his sister) on someone other than himself ("Arshia said to me to throw the shoe") in the hope of avoiding punishment. With this evidence, the clinical picture was somewhat consistent with ADHD and oppositional defiant disorder.

Imaginary friends or companions are common among all children. Children who have imaginary friends are more likely to report hearing voices. Imaginary friends disappear at the wish of the child and do not cause any threat. They can even be a source of comfort. With an imaginary friend, a child can express his true thoughts and has no need to be egodystonic. Children with imaginary friends do not typically show evidence of thought disorder (15).

When considering the differential diagnosis of hallucinations, a clinician should rule out underlying causes of hallucinations, such as substance abuse, physical disorders, and central nervous system involvement $(2,14)$. The child in our study was evaluated for these causes, and all of them were ruled out.

To precisely diagnose hallucinations in children, clinicians need a broad clinical perspective. Unfamiliarity with the phenomenon of non-psychotic hallucinations can promote the tendency for a clinician to look for a more familiar diagnosis that fits the patient's presentation, even if all clinical criteria are not fulfilled $(16,17)$.

Associated behavior involved in the presentation might be helpful, to some extent, in differentiating be- tween psychotic and non-psychotic hallucinations. Children with non- psychotic hallucinations do not present symptoms of delusional beliefs, disturbed language production, diminished motor activity, incongruous mood, bizarre behavior, or social withdrawal (7). The child in this study did not show any obvious evidence of psychotic disorder, communicated well, and answered the interviewer's questions. Thus, his hallucinations are likely non-psychotic.

DSM-IV-TR and even DSM-V do not address the clinical phenomenon of non-psychotic hallucinations in children. Accordingly, Garralda's concept of non-psychotic hallucinations in children is used in this article $(2,7,18,19)$.

Kelleher and colleagues, in a study conducted in 2012 on 2,243 children, found that psychotic symptoms are prevalent in a wide range of non-psychotic psychopathologies. The majority of children in the study who reported psychotic symptoms had at least one diagnosable psychiatric disorder not related to a particular diagnosis. Rather, a variety of Axis I disorders were associated with psychotic symptoms (8). This was also true in our case, and different Axis I disorders were considered as diagnoses. However, the question arises, why might a wide range of psychiatric disorders in children be associated with psychotic symptoms? It is likely that the same underlying risk factors may predispose to both of them (8). Although psychosis has conventionally been considered separate from neurotic disorders, a number of researchers are doubtful about the independence of psychoses and non-psychotic psychopathology (20-22).

In this case, there is a possibility that, because of the similar psychopathology between neurotic and psychosis, the hallucinations of the child in our study were associated with anxiety. This possibility raises the question of why olanzapine and risperidon were used in the treatment of this child. The answer is that, despite educational interventions, the child's aggression did not dissipate, and the child/parent interaction impairment resulting from the child taking these drugs was justified. Additionally, the fact that the boy's hallucinations never ceased is further proof that the hallucinations were non-psychotic.

Biological, psychological, and social complex creates a path for better intervention as the result of further understanding of psychosis. In view of current discoveries and scientific advances, psychosis is not seen as a wholesome biogenetic disease (23). Hearing voices or having paranoid thoughts are experiences that are often a response to trauma, abuse, or hardship (24). Thus, it appears that stress, abuse, and emotional wounds contributed to our patient's hallucinations. As stated by Longden, this cause does not rule out the use of medication. Rather, medication is one of a number of possible integrated inter- 
ventions, including psychological, social, and emotionally restorative (25).

Although the association between childhood trauma and psychosis is supported by robust literature, the psychological mechanism is poorly understood. Varese showed that dissociative experiences mediate between childhood trauma and hallucinations (26). Further, according to Morrison's approach, trauma can alter a child's beliefs about the self, the world, and others (27). As evidence of this concept, the child in this study considered himself vulnerable, others as untrustworthy (e.g., his family members were not allowed to touch his belongings), and the world as unsafe (e.g., the child did not like to leave the house).

Childhood trauma may impress psychological and neurobiological development, leading to an extensive range of symptoms and disorders comprising psychosis (28). Trauma in childhood is affiliated with a range of mental health problems, including major depression, posttraumatic stress disorder, addiction, and psychosis. Being abused as a child increases one's risk of behavioral dyscontrol, delinquency, and a lifetime history of aggression (29). In this case, prevention programs that aim to improve the parents' control over their physical aggression and their parenting skills deserve serious consideration.

The strongest facet of this report is that we studied this child prospectively over three years. Additionally, he was admitted to a hospital that is the psychiatric hub of the southeast of the country and is one of the few high-quality mental hospitals in the entire country. Moreover, after leaving the hospital, his treatment continued with a child and adolescent psychiatrist. It is further recommended that this child remain under supervision for an extended period of time.

Our research indicates that no study has been conducted on this issue in Iran. Moreover, there seems to be a lack of investigation into children's sustained hallucinations over a long period of time, especially when the probability of non-psychotic disorders is discussed. Even if the disorder of the child in our study proves to be psychotic, no significant changes were seen after treatment. It is likely that his everyday exposure to trauma caused his persistent hallucinations and aggression.

The findings of this case study illustrate that inquiries about early adversity should be routine during the assessment and treatment of psychosis. Clinical formulations and treatment plans should consider the discoveries from these inquiries. It is expected that this report will pave the way for further studies in this field.

\section{Footnote}

Authors' Contribution: The patient in this case report visited and followed up by first author. The second author gathered information, prepared the case report, and submitted it for publication.

\section{References}

1. Sadock BJ, Sadock VA. Kaplan \& Sadock's concise textbook of clinical psychiatry. Lippincott Williams \& Wilkins; 2008.

2. Edelsohn GA. Hallucinations in children and adolescents: considerations in the emergency setting. Am J Psychiatry. 2006;163(5):781-5. doi: 10.1176/ajp.2006.163.5.781. [PubMed:16648315].

3. Schreier HA. Hallucinations in nonpsychotic children: more common than we think? JAm Acad Child Adolesc Psychiatry. 1999;38(5):6235. doi: 10.1097/00004583-199905000-00028. [PubMed: 10230196].

4. Yates TT, Bannard JR. The "haunted" child: grief, hallucinations, and family dynamics. J Am Acad Child Adolesc Psychiatry. 1988;27(5):573-81. doi: 10.1097/00004583-198809000-00010. [PubMed: 3182621].

5. Kotsopoulos S, Kanigsberg J, Cote A, Fiedorowicz C. Hallucinatory experiences in nonpsychotic children. J Am Acad Child Adolesc Psychiatry. 1987;26(3):375-80. doi: 10.1097/00004583-198705000-00017. [PubMed: 3597292].

6. American Psychiatric Association . Diagnostic and statistical manual of mental disorders. 5th ed. American psychiatric publishing; 2013.

7. Garralda ME. Characteristics of the psychoses of late onset in children and adolescents (a comparative study of hallucinating children). $J$ Adolesc. 1985;8(2):195-207. [PubMed: 4019879].

8. Kelleher I, Keeley H, Corcoran P, Lynch F, Fitzpatrick C, Devlin N, et al. Clinicopathological significance of psychotic experiences in non-psychotic young people: evidence from four population-based studies. BrJ Psychiatry. 2012;201(1):26-32. doi: 10.1192/bjp.bp.111.101543. [PubMed: 22500011].

9. van Os J, Hanssen M, Bijl RV, Vollebergh W. Prevalence of psychotic disorder and community level of psychotic symptoms: an urbanrural comparison. Arch Gen Psychiatry. 2001;58(7):663-8. [PubMed: 11448373].

10. Bebbington PE, Bhugra D, Brugha T, Singleton N, Farrell M, Jenkins $\mathrm{R}$, et al. Psychosis, victimisation and childhood disadvantage: evidence from the second British National Survey of Psychiatric Morbidity. Br J Psychiatry. 2004;185:220-6. doi: 10.1192/bjp.185.3.220. [PubMed: 15339826].

11. Shevlin M, Houston JE, Dorahy MJ, Adamson G. Cumulative traumas and psychosis: an analysis of the national comorbidity survey and the British Psychiatric Morbidity Survey. Schizophr Bull. 2008;34(1):193-9. doi:10.1093/schbul/sbm069. [PubMed: 17586579].

12. Dvir Y, Denietolis B, Frazier JA. Childhood trauma and psychosis. Child Adolesc Psychiatr Clin N Am. 2013;22(4):629-41. doi: 10.1016/j.chc.2013.04.006. [PubMed: 24012077].

13. Sidhu KAS, Dickey III TO. Hallucinations in children: Diagnostic and treatment strategies. Curr Psychiatr. 2010;9:54-61.

14. Pao M, Lohman C, Gracey D, Greenberg L. Visual, tactile, and phobic hallucinations: recognition and management in the emergency department. Pediatr Emerg Care. 2004;20(1):30-4. [PubMed: 14716163].

15. Poulton R, Caspi A, Moffitt TE, Cannon M, Murray R, Harrington H. Children's self-reported psychotic symptoms and adult schizophreniform disorder: a 15-year longitudinal study. Arch General Psychiatr. 2000;57(11):1053-8.

16. Perera H, Attygalle U, Jeewandara C, Jayawardena V. Non-psychotic auditory hallucinations in children and adolescents. Sri Lanka JPsychiatr. 2011;2(1).

17. Kamaldeep Bhui DB. Clinical management of patients across cultures. Adv Psychiatr Treat. 1997;3:233-9. 
18. American Psychiatric Association . Diagnostic and Statistical manual of mental disorders. ; 2000

19. American Psychiatric Association . Diagnostic and Statistical Manual of Mental Disorders. Text Revision. Washington: American Psychiatric Association; 2013.

20. Garety PA, Kuipers E, Fowler D, Freeman D, Bebbington PE. A cog nitive model of the positive symptoms of psychosis. Psychol Med. 2001;31(2):189-95. [PubMed: 11232907].

21. Freeman D, Garety PA. Connecting neurosis and psychosis: the direct influence of emotion on delusions and hallucinations. Behav Res Ther 2003;41(8):923-47. [PubMed: 12880647].

22. Freeman D, Garety PA, Kuipers E, Fowler D, Bebbington PE. A cognitive model of persecutory delusions. Br J Clin Psychol. 2002;41(4):331-47.

23. Read J, Bentall RP, Fosse R. Time to abandon the bio-bio-bio model of psychosis: Exploring the epigenetic and psychological mechanisms by which adverse life events lead to psychotic symptoms. Epidemiol Psichiatr Soc. 2009;18(4):299-310. [PubMed: 20170043].

24. British Psychological Society . Understanding psychosis and schizophrenia. A report by the Division of Clinical Psychology. Leicester: British Psychological Society; 2014.

25. Longden E, Read J. Social Adversity in the Etiology of Psychosis: A Review of the Evidence. Am J Psychother. 2016;70(1):5-33. [PubMed: 27052604].

26. Varese F, Barkus E, Bentall RP. Dissociation mediates the relationship between childhood trauma and hallucination-proneness. Psychol Med. 2012;42(5):1025-36. doi: 10.1017/S0033291711001826. [PubMed: 21896238].

27. Morrison AP. The interpretation of intrusions in psychosis: an integrative cognitive approach to hallucinations and delusions. Behav Cogn Psychother. 2001;29(03):257-76.

28. Read J, Fosse R, Moskowitz A, Perry B. The traumagenic neurodevelopmental model of psychosis revisited. Neuropsychiatry. 2014;4(1):65-79.

29. DeJong J, Virkkunen M, Linnoila M. Factors associated with recidivism in a criminal population. J Nerv Ment Dis. 1992;180(9):543-50. [PubMed: 1522403]. 